\title{
QUANTUM PHYSICS \\ AND \\ ORDINARY LANGUAGE
}




\title{
QUANTUM PHYSICS \\ AND \\ ORDINARY LANGUAGE
}

\author{
T. BERGSTEIN
}


ISBN 978-0-333-18227-7 ISBN 978-1-349-02590-9 (eBook)

DOI 10.1007/978-1-349-02590-9

(C) T. Bergstein, 1972

Reprint of the original edition 1972

All rights reserved. No part of this publication may be reproduced or transmitted in any form or by any means, without permission

First published 1972

Paperback edition published 1974

THE MACMILLAN PRESS LTD

London and Basingstoke

Associated companies in New York Melbourne

Dublin Johannesburg and Madras

SBN 333141563 


\section{PREFACE}

This book deals with the interdependence of the phenomenological and conceptual levels of experience. This interdependence is most objectively demonstrated by the complementarity of quantum physics. When examining the basic function of ordinary language within quantum physics it is seen that complementarity is fundamental to human comprehension itself, particularly to the interaction of language and mind.

I wish to express my gratitude to Professor L. Rosenfeld, Nordic Institute for Theoretical Atomic Physics, Copenhagen. Professor Rosenfeld has revised the manuscript very carefully and has been extremely helpful in solving crucial problems of formulation.

T. Bergstein

Aars, January 1972 
What the philosophers say about reality is often just as deceptive as when, in a second-hand shop, you come across a signboard: Mangling done. If you brought your linen to have it mangled, you would be deceived; for the signboard is just for sale.

(Søren Kierkegaard) 


\section{CONTENTS}

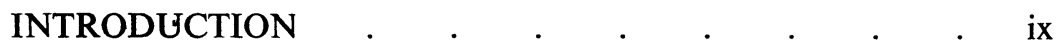

1. REMARKS ON THE CONCEPTUAL FOUNDATIONS OF SCIENCE . . . . . . . . . . . . . 1

2. ON THE FOUNDATIONS OF QUANTUM PHYSICS . . 7

3. THE PRINCIPLE OF COMPLEMENTARITY . . . 19

4. QUANTUM PHYSICS AND ORDINARY LANGUAGE . 27

5. LOGIC AND COMPLEMENTARITY . . . . 38

6. PHILOSOPHY AND COMPLEMENTARITY . . . . 41

7. LANGUAGE AND MIND . . . . . . . 53 


\section{INTRODUCTION}

Language has become one of the most important objects of philosophy. Of course language has always been the necessary medium of philosophical inquiry, but modern philosophers have focused their attention on language itself, assuming that experiences that can be comprehended only through language must be inseparable from language. Doubts have been raised against the conception of traditional philosophy that the primary function of language is to describe a world of phenomena, which have an existence independent of langauge, and one begins to perceive that the problem of language and reality is related to that of the human mind and the external world. It seems likely that language and human individuality mutually confirm each other, and it is a basic epistemological problem to study their development and interaction.

The problems of the significance of the conceptual frame in modern linguistic philosophy and in physical science exhibit remarkable similarities, suggesting that an intensified communication between linguistic philosophers and theoretical physicists might be profitable. Undoubtedly this proposition will provoke severe opposition on both sides. Many philosophers will claim that true philosophy deals with statements that can be neither verified nor refuted by natural science, and a majority of scientists will say that ordinary language is a means of communication so inaccurate that it can have no fundamental significance to natural science. But modern physics has shown incontestably that the conceptual framework of natural science is ultimately based on ordinary language. The reason why so little attention is paid to this condition, even among physicists, is that current scientific work is always performed within a given conceptual frame, the validity of the axiomatic foundation of this frame being taken for granted. To consider the construction of a new or more general conceptual frame is indeed an extremely difficult task, which only rare geniuses like Newton and Bohr can achieve. But naturally this does not prevent philosophers from taking into account the epistemological significance of general physical theories already available, especially when they affect fundamental concepts of ordinary language.

Philosophers often put forward the opinion that scientific results cannot be significant to general philosophy because they may sooner or later turn out to be false. But since the time of Galileo physical science has been based on measurements, and physical description has been concerned 
with the simplest possible linking together of the greatest possible number of experimental results. Results of measurements which can be reproduced as often as required are incontestable, and are thus the unifications of descriptions obtained theoretically. Presumably the timeless character of fundamental physical concepts, principles, and laws is hard for many philosophers to accept because they confuse temporariness with possible limitations in the range of applicability. But in order to judge the epistemological significance of a limitation in range of applicability of a fundamental physical concept, it is essential to observe that the discovery of such a limitation always happens through some remarkable advance in experimental technique, and that the limit of applicability coincides with a split between phenomena observable by the usual means of observation and phenomena that can be observed only by the new technique. Hence it is perfectly possible that the physical concept in question can preserve its fundamental function in physical description, and the very limitation in range of applicability may serve as a starting point for the construction of a more general theory.

From the historical point of view it is evident that science and philosophy are intimately interdependent. The history of European cultures shows that these manifestations of human thought are so closely related that the one could not have come to existence without the other. Modern natural science is the outcome of natural philosophy that was generated by the introduction of empirical methods of investigation and systematic classification of phenomena in philosophical inquiry. But, as already indicated, there are far more momentous arguments showing the interdependence of natural science and other aspects of human thinking. The discoveries in this century have forced physicists to analyse the conceptual foundation of science itself, and it has become evident that it is deeply anchored in the groundwork of human cognition. Modern quantum physics has demonstrated that science and epistemology join in the substratum of ordinary language that supports axiomatically a definite classification of external phenomena. This conjunction establishes the possibility of mutual influence, but of course epistemological significance is not to be found in the result of every piece of scientific research. An interaction is only possible when scientific experience necessitates a change in some part of the axiomatic foundation of the conceptual frame.

The common axiomatic basis of natural science and epistemology before quantum physics consisted mainly in an idealization of the part of ordinary language dealing with the material objects and events of the external world, and the change introduced by quantum physics affects such fundamental concepts as space, time, and causality. It is a crucial point, however, that the ordinary conception and use of the words are not contested. On the contrary the immediate validity of ordinary language is 
still fundamental to physical description. The contest is about the idealization of language introduced in classical physical theories as well as in classical philosophical problems. In ordinary language, concepts treating spatial, temporal, and causal conditions can be used without mutual restrictions other than those imposed by logic and normal syntax, and this freedom is fundamental for the unambiguous separation of space and time as well as for the division of experiences into a private world and an external world. Mainly by using mathematical abstractions, classical physics has idealized the conceptions of space, time, and causality, tacitly assuming that there are no mutual restrictions in the applicability of the concepts. But quantum physics has shown incontestably that such restrictions do exist.

Unfortunately, natural philosophy seems to be fixed in the conceptual frame of classical physics. Certainly, quantum physics is often referred to in epistemological investigations, but few professional philosophers have been able to free themselves from the idealization of dynamics that penetrates classical philosophy. Hence, there must be some kind of ambiguity in the attitude of natural philosophy towards modern science. The enormous advance in natural science in this century has brought to mankind a knowledge of nature which lies far beyond the aims of classical philosophy. Modern physics has made it possible to describe material phenomena of the greatest diversity as combinations and interactions of the elementary particles observed in atomic and nuclear physics. This unification of the material world resulting from incontestable experimental discoveries has left a vacuum in the traditional field of philosophical inquiry. The extensive literature of modern natural philosophy is a paradoxical product of the situation. It does not express any advance in human knowledge which is not already available in scientific papers. On the contrary the main part of this literature contains erroneous interpretations of basic physical theories. The cause of this unfortunate situation must be sought in the circumstance that the conceptual frame of modern physics cannot be comprehended in any philosophical language or terminology based on the traditional ontological way of thinking. Yet it is possible to consider modern quantum theory as the physical representative of a fundamental mode of cognition which has the greatest significance not only for philosophy but for every kind of human thinking. This mode of cognition refers to a basic condition to which every kind of observation and communication is submitted. It was in fact perceived thousands of years ago by the philosophers of ancient India and China. In modern science Niels Bohr has given it the name of complementarity.

The purpose of this book is to make the relation of complementarity more widely known and to show how it is indispensable to human comprehension and communication of experience. In order to describe comple- 
mentarity unambiguously and to base the argumentation on solid ground, the first chapters deal with the foundations of classical and quantum physics and with their interdependence. The presentation is also intended to show the important function of ordinary language in the unambiguous interpretation of physical concepts; therefore the physical content of any employed mathematical symbol will be properly explained in ordinary language. This presentation should not be considered as a popularization of modern physics, but rather as an analysis adequate to the subject under consideration. It is based on the fundamental assumption that ordinary language is the most comprehensive means of communication. 